\title{
SUPPORTING INFORMATION \\ Hierarchical propagation of chirality through reversible polymerization: the cholesteric phase of DNA oligomers
}

\author{
Cristiano De Michele, ${ }^{1, *}$ Giuliano Zanchetta, ${ }^{2}$ Tommaso Bellini, ${ }^{2}$ Elisa Frezza, ${ }^{3, \dagger}$ and Alberta Ferrarini ${ }^{3}$ \\ ${ }^{1}$ Dipartimento di Fisica, "Sapienza" Università di Roma, P.le A. Moro 2, 00185 Roma, Italy \\ ${ }^{2}$ Dipartimento di Biotecnologie Mediche e Medicina Traslazionale, \\ Università di Milano, via F.lli Cervi 93, Segrate (MI) Italy \\ ${ }^{3}$ Dipartimento di Scienze Chimiche, Università di Padova, via Marzolo 1, I-35131 Padova, Italy
}

(Dated: December 22, 2015)

The first section reports experiment details. The next section outlines the theoretical approach that we have developed to calculate the free energy and chiral properties of self-assembly-driven cholesteric $\left(\mathrm{N}^{*}\right)$ liquid crystals. The final section summarizes the parameterization used in calculations for Dickerson dodecamers.

\section{EXPERIMENTAL METHODS}

Sample preparation and concentration measurement

Liquid crystal samples were prepared by loading a drop of the sample solution between two glass slides separated by 20 micrometers polymer film spacers, then slowly evaporated until the final concentration was reached and sealed with a fluorinated oil; the sample was then thermally cycled to let the DNA concentration homogenize. To measure concentration, the sample was brought, through heating, into the isotropic phase where the spectrum of the reflected intensity was measured in a microscope. The frequency of the multiple reflection fringes due to the interference from the parallel cell plates, was systematically measured in various spots within the DNA solution and on the periphery of the cells, where the fluorinated oil was instead present. This set of data enabled determining the exact thickness of the cell, the refractive index of the DNA solution and thus the DNA concentration through a calibration curve.

\section{Characterization of the $\mathrm{N}^{*}$ phase}

Microscopic observations were made either on a Nikon TE200 or a Nikon Optiphot2 microscope, equipped with a Nikon DS-5M camera and an Instec MK2 hot stage. The average $\mathrm{N}^{*}$ pitch $p$ was estimated from Fourier analysis of polarized microscopy images of the so-called fingerprint textures of the $\mathrm{N}^{*}$ phase [1]. When the pitch of the $\mathrm{N}^{*}$ phase is in the $\mu \mathrm{m}$ range, measurements of the transmitted intensity though planar textures as a function of the analyzer position enable determining the optical rotation, in turn related to the handedness of the phase. The rotatory power $\rho$, defined as the angle of rotation per unit length - where positive $\rho$ means a left-handed rotation - is simply expressed as a function of the basic optical properties of the $\mathrm{N}^{*}$ structure. In the case of $\mathrm{N}^{*}$ pitch longer than visible wavelength (with $p>2 \lambda$ and $p \Delta n \ll \lambda$, where $\Delta n$ is the optical birefringence whose typical values for DNA cholesterics are between 0.02 and 0.04 ), the optical rotation is well approximated by [2]:

$$
\rho=-\left(\pi * \Delta n^{2} * p\right) / 4 \lambda^{2}
$$

\section{THEORY}

\section{Free energy of the system}

The system under investigation is a cholesteric phase of pitch $p$, or wavenumber $q=2 \pi / p$, formed by a polydisperse mixture of self-assembling linear aggregates. Each aggregate is formed by $l$ monomers $(1 \leq l \leq \infty)$; the total number of monomers is $N, v_{m}$ is the volume of a monomer and $V$ the total volume. Therefore $\rho=N / V$ is the number density of monomers and $\phi=\rho v_{m}$ is the volume fraction of monomers. We can then define the monomer density function $\bar{\rho}(l, \boldsymbol{\Omega})$, where $l$ is the length of the aggregate to which a monomer belongs and $\boldsymbol{\Omega}$ is the set of Euler angles which define the monomer orientation in a laboratory $(\mathrm{LAB})$ frame. It obeys the normalization condition $\sum_{l=1}^{\infty} \int d \boldsymbol{\Omega} \bar{\rho}(l, \boldsymbol{\Omega})=\rho$. 
We can write $\bar{\rho}(l, \boldsymbol{\Omega})=l \nu(l) f_{l}(\boldsymbol{\Omega})$, where $\nu(l)$ is the number density of aggregates of length $l$, normalized such that $\sum_{l=1}^{\infty} l \nu(l)=\rho$, and $f_{l}(\boldsymbol{\Omega})$ is the orientational distribution function of aggregates of length $l$. This is normalized as $\int d \boldsymbol{\Omega} f_{l}(\boldsymbol{\Omega})=1$ and in the isotropic phase is equal to a constant.

The Helmholtz free energy of the system is expressed as a functional of the monomer density function $\bar{\rho}(l, \boldsymbol{\Omega})$, and is written as the sum of a number of contributions as follows:

$$
F[\bar{\rho}(l, \boldsymbol{\Omega})]=F^{i d}+F^{s t}+F^{o r}+F^{e x c l}
$$

where $F^{i d}$ is the ideal gas term, $F^{s t}$ is a term relative to stacking interactions, $F^{\text {or }}$ describes the entropy decrease due to orientational order in the liquid crystal phase and $F^{e x c l}$ accounts for excluded volume interactions between aggregates. The first term is simply given by [3]:

$$
\frac{F^{i d}}{k_{B} T V}=\sum_{l=1}^{\infty} \nu(l)\left\{\ln \left[v_{m} \nu(l)\right]-1\right\}
$$

where $k_{B}$ is the Boltzmann constant and $T$ is the temperature, whereas the stacking free energy can be expressed as $[4,5]$ :

$$
\frac{F^{s t}}{k_{B} T V}=-\frac{\Delta F_{b}}{k_{B} T} \sum_{l=1}^{\infty}(l-1) \nu(l)
$$

with $-\Delta F_{b}$ being the free energy gain associated to the stacking interaction between a pair of monomers. The orientational contribution to the free energy can be expressed in the form [4]:

$$
\frac{F^{o r}}{k_{B} T V}=\hat{\sigma}_{o} \equiv \sum_{l=1}^{\infty} \sigma_{o}(l) \nu(l)
$$

where $\sigma_{o}(l)$ is the entropy that a chain of length $l$ looses in the liquid crystal phase. The form of the two excluded volume contribution in Eq. (2) will be discussed in the following.

It is worth noting that in the free energy of Eq. (2) the chain length distribution $\nu(l)$ and the orientational order, described by the distribution function $f_{l}(\boldsymbol{\Omega})$, are coupled. Therefore, the equilibrium state and the chiral organization of the system are determined by the mutual interplay of aggregation and order.

\section{Excluded volume contribution}

In the following, we will first derive analytic expressions for the excluded volume contribution to the free energy for the case of linear aggregates. This can be conveniently estimated through direct numerical integration, given a specific model for the aggregates. Subsequently, a scaling argument will be used to express the excluded volume contribution to the free energy of semi-flexible aggregates in terms of the forms obtained for stiff aggregates.

\section{Linear aggregates}

Let us start considering a mixture of hard linear aggregates of fixed length, with $N_{l}$ being the number of aggregates of length $l$. The excluded volume contribution to the free energy density can be expressed as:

$$
\frac{F^{e x c l}}{V}=\frac{k_{B} T \eta(\phi)}{2} \sum_{l} \sum_{l^{\prime}} \nu(l) \nu\left(l^{\prime}\right) \bar{v}^{e x c l}\left(l, l^{\prime}\right)
$$

where $\nu(l)=\left(N_{l} / V\right)$ is the number density of polymers of length $l, \eta(\phi)=(1 / 4)(4-3 \phi) /(1-\phi)^{2}$ is the Parsons-Lee factor, which corrects for the neglect of higher virial contributions [6-8] and $\bar{v}^{\text {excl }}\left(l, l^{\prime}\right)$ is the volume excluded to an aggregate of length $l^{\prime}$ by an aggregate of length $l$, integrated over all their orientations:

$$
\bar{v}^{e x c l}\left(l, l^{\prime}\right)=-\frac{1}{V} \int d \mathbf{R}_{1} d \mathbf{R}_{2} d \boldsymbol{\Omega}_{1} d \boldsymbol{\Omega}_{2} e_{12}^{l l^{\prime}}\left(\mathbf{R}_{12}, \boldsymbol{\Omega}_{1}, \boldsymbol{\Omega}_{2}\right) f_{l}\left(\boldsymbol{\Omega}_{1}\right) f_{l^{\prime}}\left(\boldsymbol{\Omega}_{2}\right)
$$


Here $\mathbf{R}_{i}$ is the vector position of the $i$ aggregate and $\boldsymbol{\Omega}_{i}$ are the Euler angles that define its orientation in a laboratory (LAB) frame; $\mathbf{R}_{12}=\mathbf{R}_{2}-\mathbf{R}_{1}$ is the relative position of 2 with respect to 1 , and $e_{12}^{l l^{\prime}}$ is the Mayer function [3]:

$$
e_{12}^{l l^{\prime}}\left(\mathbf{R}_{12}, \boldsymbol{\Omega}_{1}, \boldsymbol{\Omega}_{2}\right)=\exp \left\{-U_{h}\left(\mathbf{R}_{12}, \boldsymbol{\Omega}_{1}, \boldsymbol{\Omega}_{2}\right) / k_{B} T\right\}-1
$$

with $U_{h}\left(\mathbf{R}_{12}, \boldsymbol{\Omega}_{1}, \boldsymbol{\Omega}_{2}\right)$ being the hard-core pair potential:

$$
U_{h}\left(\mathbf{R}_{12}, \boldsymbol{\Omega}_{1}, \boldsymbol{\Omega}_{2}\right)= \begin{cases}\infty & \text { if } 1,2 \text { overlap } \\ 0 & \text { if } 1,2 \text { do not overlap. }\end{cases}
$$

In principle the orientational distribution function, $f_{l}(\boldsymbol{\Omega})$, in the $\mathrm{N}^{*}$ phase depends on both the degree of orientational order and the helical pitch $p$. However, since the latter is orders of magnitude longer than the molecular scale we can safely assume $f_{l}(\boldsymbol{\Omega})$, taken with respect to the local director, to be the same as in the corresponding untwisted $\mathrm{N}$ phase $(p \rightarrow \infty)$. This assumption is justified also in the case of very long aggregates, since the characteristic length scale of molecular chirality is of the order of the nanometer, whereas the $\mathrm{N}^{*}$ pitch is in the micrometer range. Thus, for rod-like aggregates in the untwisted $\mathrm{N}$ phase we can write $f_{l}(\boldsymbol{\Omega})=f_{l}(\cos \theta)$, with $\cos \theta=\hat{\mathbf{n}} \cdot \hat{\mathbf{u}}$, where $\hat{\mathbf{u}}$ and $\hat{\mathbf{n}}$ are unit vectors parallel to the axis of the helical aggregate and to the director, respectively. Since the $\mathrm{N}^{*}$ pitch is expected to be much larger than particle size, one can express the orientational distribution function of a particle at the position $\mathbf{R}_{2}=\mathbf{R}_{1}+\mathbf{R}_{12}$ in terms of that of particle at the position $\mathbf{R}_{1}\left(\left\|\mathbf{R}_{12}\right\| \ll p\right)$ by the following Taylor expansion truncated to second order:

$$
\begin{aligned}
& f_{l}\left(\hat{\mathbf{u}} \cdot \hat{\mathbf{n}}\left(\mathbf{R}_{2}\right)\right) \approx f_{l}\left(\hat{\mathbf{u}} \cdot \hat{\mathbf{n}}\left(\mathbf{R}_{1}\right)\right)+R_{12}^{\alpha}\left[\partial_{R^{\alpha}} f_{l}(\hat{\mathbf{u}} \cdot \hat{\mathbf{n}}(\mathbf{R}))\right]_{\mathbf{R}=\mathbf{R}_{1}}+\frac{1}{2} R_{12}^{\alpha} R_{12}^{\beta}\left[\partial_{R^{\alpha}} \partial_{R^{\beta}} f_{l}(\hat{\mathbf{u}} \cdot \hat{\mathbf{n}}(\mathbf{R}))\right]_{\mathbf{R}=\mathbf{R}_{1}} \\
& =f_{l}\left(\hat{\mathbf{u}} \cdot \hat{\mathbf{n}}\left(\mathbf{R}_{1}\right)\right)+\left.R_{12}^{\alpha} \dot{f}_{l}(x)\right|_{x=\hat{\mathbf{u}} \cdot \hat{\mathbf{n}}\left(\mathbf{R}_{1}\right)}\left[\partial_{R^{\alpha}}(\hat{\mathbf{u}} \cdot \hat{\mathbf{n}}(\mathbf{R}))\right]_{\mathbf{R}=\mathbf{R}_{1}} \\
& +\frac{1}{2} R_{12}^{\alpha} R_{12}^{\beta}\left\{\partial_{R^{\alpha}}\left[\left.\dot{f}_{l}(x)\right|_{x=\hat{\mathbf{u}} \cdot \hat{\mathbf{n}}(\mathbf{R})} \partial_{R^{\beta}}(\hat{\mathbf{u}} \cdot \hat{\mathbf{n}}(\mathbf{R}))\right]\right\}_{\mathbf{R}=\mathbf{R}_{1}} \\
& =f_{l}\left(\hat{\mathbf{u}} \cdot \hat{\mathbf{n}}\left(\mathbf{R}_{1}\right)\right)+\left.R_{12}^{\alpha} \dot{f}_{l}(x)\right|_{x=\hat{\mathbf{u}} \cdot \hat{\mathbf{n}}\left(\mathbf{R}_{1}\right)} \partial_{R_{1}^{\alpha}}\left(\hat{\mathbf{u}} \cdot \hat{\mathbf{n}}\left(\mathbf{R}_{1}\right)\right)+\frac{1}{2} R_{12}^{\alpha} R_{12}^{\beta} \partial_{R_{1}^{\alpha}}\left[\left.\dot{f}_{l}(x)\right|_{x=\hat{\mathbf{u}} \cdot \hat{\mathbf{n}}\left(\mathbf{R}_{1}\right)} \partial_{R_{1}^{\beta}}\left(\hat{\mathbf{u}} \cdot \hat{\mathbf{n}}\left(\mathbf{R}_{1}\right)\right)\right]
\end{aligned}
$$

where $R^{\alpha}, R_{1}^{\alpha}$ and $R_{12}^{\alpha}$ with $\alpha=x, y, z$ are the cartesian components of the vectors $\mathbf{R}, \mathbf{R}_{1}$ and $\mathbf{R}_{12}$ respectively and the Einstein convention is used for summations over repeated indices $\alpha$ and $\beta$. Here, $\dot{f}_{l}$ is the first derivative of the function $f_{l}$ with respect to its argument, i.e. $\dot{f}_{l}=d f_{l}(x) / d x$. Using Eq. (10) to express the orientational distribution function of particle 2 in terms of that of particle 1, Eq. (7) can be rewritten as:

$$
\begin{aligned}
& \bar{v}^{e x c l}\left(l, l^{\prime}\right)=-\frac{1}{V} \int d \mathbf{R}_{1} d \mathbf{R}_{12} d \boldsymbol{\Omega}_{1} d \boldsymbol{\Omega}_{2} e_{12}^{l l^{\prime}}\left(\mathbf{R}_{12}, \boldsymbol{\Omega}_{1}, \boldsymbol{\Omega}_{2}\right) f_{l}\left(\mathbf{u}_{\mathbf{1}} \cdot \hat{\mathbf{n}}\left(\mathbf{R}_{1}\right)\right) f_{l^{\prime}}\left(\mathbf{u}_{\mathbf{2}} \cdot \hat{\mathbf{n}}\left(\mathbf{R}_{1}\right)\right) \\
& -\left.\frac{1}{V} \int d \mathbf{R}_{1} d \mathbf{R}_{12} d \boldsymbol{\Omega}_{1} d \boldsymbol{\Omega}_{2} e_{12}^{l l^{\prime}}\left(\mathbf{R}_{12}, \boldsymbol{\Omega}_{1}, \boldsymbol{\Omega}_{2}\right) f_{l}\left(\mathbf{u}_{\mathbf{1}} \cdot \hat{\mathbf{n}}\left(\mathbf{R}_{1}\right)\right) R_{12}^{\alpha} \dot{f}_{l^{\prime}}(x)\right|_{x=\hat{\mathbf{u}}_{2} \cdot \hat{\mathbf{n}}\left(\mathbf{R}_{1}\right)} \partial_{R_{1}^{\alpha}}\left(\mathbf{u}_{\mathbf{2}} \cdot \hat{\mathbf{n}}\left(\mathbf{R}_{1}\right)\right) \\
& +\left.\frac{1}{2 V} \int d \mathbf{R}_{1} d \mathbf{R}_{12} d \boldsymbol{\Omega}_{1} d \boldsymbol{\Omega}_{2} e_{12}^{l l^{\prime}}\left(\mathbf{R}_{12}, \boldsymbol{\Omega}_{1}, \boldsymbol{\Omega}_{2}\right) R_{12}^{\alpha} R_{12}^{\beta} \dot{f}_{l}(x)\right|_{x=\hat{\mathbf{u}}_{1} \cdot \hat{\mathbf{n}}\left(\mathbf{R}_{1}\right)} \partial_{R_{1}^{\alpha}}\left(\mathbf{u}_{\mathbf{1}} \cdot \hat{\mathbf{n}}\left(\mathbf{R}_{1}\right)\right) \\
& \times\left.\dot{f}_{l^{\prime}}(x)\right|_{x=\hat{\mathbf{u}}_{2} \cdot \hat{\mathbf{n}}\left(\mathbf{R}_{1}\right)} \partial_{R_{1}^{\beta}}\left(\mathbf{u}_{\mathbf{2}} \cdot \hat{\mathbf{n}}\left(\mathbf{R}_{1}\right)\right)
\end{aligned}
$$

where the last term has been obtained by integrating by parts and neglecting the surface term, which is not relevant if we are interested in bulk properties. Considering now a reference frame having the $Y$-axis along the helical axis, one can write:

$$
\hat{\mathbf{n}}\left(\mathbf{R}_{1}\right)=\cos \left(q R_{1}^{y}\right) \hat{\mathbf{Z}}+\sin \left(q R_{1}^{y}\right) \hat{\mathbf{X}}
$$

where $q$ is the helix wavenumber, which is related to the $\mathrm{N}^{*}$ pitch $p$ by $q=2 \pi / p, R^{y}$ is the component along the $\mathrm{y}$-axis of $\mathbf{R}$ and $\hat{\mathbf{X}}, \hat{\mathbf{Y}}$ and $\hat{\mathbf{Z}}$ are unit vectors along the reference frame axes. Hence, one has:

$$
\partial_{R_{1}^{\alpha}}\left(\mathbf{u} \cdot \hat{\mathbf{n}}\left(\mathbf{R}_{1}\right)\right)=\delta_{\alpha y} \mathbf{u} \cdot\left[-q \sin \left(q R_{1}^{y}\right) \hat{\mathbf{Z}}+q \cos \left(q R_{1}^{y}\right) \hat{\mathbf{X}}\right]
$$

where $\delta_{\alpha \beta}$ is the Kronecker delta. The integrands in the outer integrals (over $\mathbf{R}_{1}$ ) of Eq. (11) are independent of $\mathbf{R}_{1}$, and are conveniently calculated in a local frame having its origin at the position of particle 1 , the $Y$-axis parallel to 
the helix axis and the Z-axis parallel to the local director. So, using Eqs. (12) and (13), Eq. (11) can be rewritten in the form:

$$
\bar{v}^{e x c l}\left(l, l^{\prime}\right)=\bar{v}_{0}^{e x c l}\left(l, l^{\prime}\right)+q \bar{v}_{1}^{e x c l}(l, l)+\frac{q^{2}}{2} \bar{v}_{2}^{e x c l}\left(l, l^{\prime}\right)
$$

where the first term represents the average excluded volume in the untwisted $\mathrm{N}$ phase:

$$
\bar{v}_{0}^{e x c l}\left(l, l^{\prime}\right)=\int d \boldsymbol{\Omega}_{1} d \boldsymbol{\Omega}_{2} M_{0}\left(\boldsymbol{\Omega}_{1}, \boldsymbol{\Omega}_{2}\right) f_{l}\left(\mathbf{u}_{\mathbf{1}} \cdot \hat{\mathbf{Z}}\right) f_{l^{\prime}}\left(\mathbf{u}_{\mathbf{2}} \cdot \hat{\mathbf{Z}}\right)
$$

the second is a chiral contribution:

$$
\bar{v}_{1}^{e x c l}\left(l, l^{\prime}\right)=\left.\int d \boldsymbol{\Omega}_{1} d \boldsymbol{\Omega}_{2} M_{1}\left(\boldsymbol{\Omega}_{1}, \boldsymbol{\Omega}_{2}\right)\left(\mathbf{u}_{\mathbf{2}} \cdot \hat{\mathbf{X}}\right) f_{l}\left(\mathbf{u}_{\mathbf{1}} \cdot \hat{\mathbf{Z}}\right) \dot{f}_{l^{\prime}}(x)\right|_{x=\hat{\mathbf{u}}_{2} \cdot \hat{\mathbf{z}}}
$$

and the latter term accounts for the elastic restoring torque:

$$
\bar{v}_{2}^{e x c l}\left(l, l^{\prime}\right)=-\left.\left.\int d \boldsymbol{\Omega}_{1} d \boldsymbol{\Omega}_{2} M_{2}\left(\boldsymbol{\Omega}_{1}, \boldsymbol{\Omega}_{2}\right)\left(\hat{\mathbf{u}}_{1} \cdot \hat{\mathbf{X}}\right)\left(\hat{\mathbf{u}}_{2} \cdot \hat{\mathbf{X}}\right) \dot{f}_{l}(x)\right|_{x=\hat{\mathbf{u}}_{1} \cdot \hat{\mathbf{z}}} \dot{f}_{l^{\prime}}(x)\right|_{x=\hat{\mathbf{u}}_{2} \cdot \hat{\mathbf{z}}}
$$

These expressions contain the moments $M_{k}$ :

$$
M_{k}^{l l^{\prime}}\left(\boldsymbol{\Omega}_{1}, \boldsymbol{\Omega}_{2}\right)=\int d \mathbf{R}_{12} Y_{12}^{k}
$$

where

$$
Y_{12}^{k}=-\left(R_{12}^{y}\right)^{k} e_{12}^{l l^{\prime}}\left(\mathbf{R}_{12}, \boldsymbol{\Omega}_{1}, \boldsymbol{\Omega}_{2}\right)
$$

By substituting Eq. (14) into Eq. (6) we obtain:

$$
\frac{F^{e x c l}}{V}=\frac{F_{0}^{e x c l}}{V}+q k_{2}+\frac{q^{2}}{2} K_{22}
$$

where $F_{0}^{e x c}$ is free energy density of the untwisted $\mathrm{N}$ phase:

$$
\frac{F_{0}^{e x c l}}{V}=\frac{k_{B} T \eta(\phi)}{2} \sum_{l} \sum_{l^{\prime}} \nu(l) \nu\left(l^{\prime}\right) \bar{v}_{0}^{e x c l}\left(l, l^{\prime}\right)
$$

$k_{2}$ is the chiral strength:

$$
k_{2}=\frac{k_{B} T \eta(\phi)}{2} \sum_{l} \sum_{l^{\prime}} \nu(l) \nu\left(l^{\prime}\right) \bar{v}_{1}^{e x c l}\left(l, l^{\prime}\right),
$$

and $K_{22}$ is the twist elastic constant:

$$
K_{22}=\frac{k_{B} T \eta(\phi)}{2} \sum_{l} \sum_{l^{\prime}} \nu(l) \nu\left(l^{\prime}\right) \bar{v}_{2}^{e x c l}\left(l, l^{\prime}\right)
$$

The two latter terms in Eq. (20), although crucial for the $\mathrm{N}^{*}$ twist, give a negligible contribution to the whole free energy of the system. Hence, the isotropic-cholesteric (I-N*) phase transition can be identified with the isotropicnematic (I-N).

\section{Semi-flexible aggregates}

In the case of semi-flexible aggregates, two additional length scales come into play, i.e. the scaled persistence length $l_{p}$ and, in liquid crystal phases, a scaled effective length $l_{0}$, which is related to the former and discriminates the regimes of short $\left(l \leq l_{0}\right)$ and long chains $\left(l>l_{0}\right)[4,9]$. The former are assumed to behave as stiff chains, whereas in the 
latter case an aggregate of length $l$ is considered to be equivalent to $\left(l / l_{0}\right)$ stiff aggregates of length $l_{0}$. Based on these considerations, we split the excluded volume terms into three contributions as follows:

$$
\bar{v}_{i}^{e x c l}\left(l, l^{\prime}\right)=\theta\left(l_{0}-1-l^{\prime}\right) \theta\left(l_{0}-1-l\right) \bar{v}_{i}^{\text {short }}\left(l, l^{\prime}\right)+2 \theta\left(l_{0}-1-l\right) \theta\left(l^{\prime}-l_{0}\right) \bar{v}_{i}^{\text {mix }}\left(l, l^{\prime}\right)+\theta\left(l-l_{0}\right) \theta\left(l^{\prime}-l_{0}\right) \bar{v}_{i}^{\text {long }}\left(l, l^{\prime}\right)
$$

where $\theta(x)$ it the Heaviside step function, $i=0,1,2$ and the upper script is use to distinguish the cases where both chains are short (long), or both are long (short), or one is long and the other short (mixed). For short chains, using Eqs. (15)-(17), we can write: $\bar{v}_{i}^{\text {short }}\left(l, l^{\prime}\right)=\bar{v}_{i}^{\text {excl }}\left(l, l^{\prime}\right)$. For long chains the following expression has been assumed: $\nu(l) \nu\left(l^{\prime}\right) \bar{v}_{i}^{\text {long }}\left(l, l^{\prime}\right)=\nu_{0}(l) \nu_{0}\left(l^{\prime}\right) c_{i}^{\text {long }}$, where $\nu_{0}(l)=\left(l / l_{0}\right) \nu(l)$ and $c_{i}^{\text {long }}$ with $i=0,1,2$ is a constant. Thus, one obtains:

$$
\bar{v}_{i}^{\text {long }}\left(l, l^{\prime}\right)=\frac{l l^{\prime}}{l_{0}^{2}} c_{i}^{\text {long }}
$$

As will be shown below, Eqs.(45)-(47), for stiff aggregates one has:

$$
\begin{aligned}
& \frac{\bar{v}_{0}^{\text {stiff }}\left(l, l^{\prime}\right)}{l l^{\prime}}=\mathcal{O}(1) \\
& \frac{\bar{v}_{1}^{\text {stiff }}\left(l, l^{\prime}\right)}{\left(l+l^{\prime}\right) / 2}=\mathcal{O}(1) \\
& \frac{\bar{v}_{2}^{\text {stiff }}\left(l, l^{\prime}\right)}{\left(l l^{\prime}\right)^{2}}=\mathcal{O}(1)
\end{aligned}
$$

so that, to fulfill the scaling laws in Eq. (25) we have assumed:

$$
\begin{array}{r}
\bar{v}_{0}^{\text {long }}\left(l, l^{\prime}\right)=\bar{v}_{0}^{\text {stiff }}\left(l, l^{\prime}\right) \\
\bar{v}_{1}^{\text {long }}\left(l, l^{\prime}\right)=\frac{2 l l^{\prime}}{l_{0}\left(l+l^{\prime}\right)} \bar{v}_{1}^{\text {stiff }}\left(l, l^{\prime}\right) \\
\bar{v}_{2}^{\text {long }}\left(l, l^{\prime}\right)=\frac{l_{0}^{2}}{l l^{\prime}} \bar{v}_{2}^{\text {stiff }}\left(l, l^{\prime}\right)
\end{array}
$$

Analogously, we have assumed for the mixed terms:

$$
\begin{aligned}
& \bar{v}_{0}^{\text {mix }}\left(l, l^{\prime}\right)=\bar{v}_{0}^{\text {stiff }}\left(l, l^{\prime}\right) \\
& \bar{v}_{1}^{\text {mix }}\left(l, l^{\prime}\right)=\frac{l^{\prime}}{l_{0}} \frac{l+l_{0}}{l+l^{\prime}} \bar{v}_{1}^{\text {stiff }}\left(l, l^{\prime}\right) \\
& \bar{v}_{2}^{\text {mix }}\left(l, l^{\prime}\right)=\frac{l_{0}}{l^{\prime}} \bar{v}_{2}^{\text {stiff }}\left(l, l^{\prime}\right)
\end{aligned}
$$

According to the above scaling laws we are now in a position to explicitly write the excluded volume in Eq. (24) in terms of the excluded volume of stiff chains $v_{i}^{\text {stiff }}\left(l, l^{\prime}\right)$. For the free energy of the untwisted $\mathrm{N}$ phase $F_{0}^{\text {excl}}$, the three terms have the same form, i.e. $v_{0}^{\text {stiff }}\left(l, l^{\prime}\right)$, so Eq. (21) is recovered also in the case of semi-flexible aggregates. For $k_{2}$ use of Eqs. (30) and (33) into Eq. (24) yields:

$$
\begin{aligned}
k_{2} & =\frac{k_{B} T \eta(\phi)}{2}\left\{\sum_{l=1}^{l_{0}-1} \sum_{l^{\prime}=1}^{l_{0}-1} \nu(l) \nu\left(l^{\prime}\right) \bar{v}_{1}^{s t i f f}\left(l, l^{\prime}\right)+2 \sum_{l=1}^{l_{0}-1} \sum_{l^{\prime}=l_{0}}^{\infty} \frac{l\left(l^{\prime}+l_{0}\right)}{l_{0}\left(l+l^{\prime}\right)} \nu(l) \nu\left(l^{\prime}\right) \bar{v}_{1}^{\text {stiff }}\left(l, l^{\prime}\right)+\right. \\
& \left.\sum_{l=l_{0}}^{\infty} \sum_{l^{\prime}=l_{0}}^{\infty} \frac{2 l l^{\prime}}{l_{0}\left(l+l^{\prime}\right)} \nu(l) \nu\left(l^{\prime}\right) \bar{v}_{1}^{\text {stiff }}\left(l, l^{\prime}\right)\right\}
\end{aligned}
$$


whereas plugging Eqs. (31) and (34) into Eq. (24) for $K_{22}$ the result is:

$$
\begin{aligned}
& K_{22}=\frac{k_{B} T \eta(\phi)}{2}\left\{\sum_{l=1}^{l_{0}-1} \sum_{l^{\prime}=1}^{l_{0}-1} \nu(l) \nu\left(l^{\prime}\right) \bar{v}_{2}^{\text {stiff }}\left(l, l^{\prime}\right)+2 \sum_{l=1}^{l_{0}-1} \sum_{l^{\prime}=l_{0}}^{\infty} \frac{l_{0}}{l^{\prime}} \nu(l) \nu\left(l^{\prime}\right) \bar{v}_{2}^{\text {stiff }}\left(l, l^{\prime}\right)+\right. \\
& \left.\sum_{l=l_{0}}^{\infty} \sum_{l^{\prime}=l_{0}}^{\infty} \frac{l_{0}^{2}}{l l^{\prime}} \nu(l) \nu\left(l^{\prime}\right) \bar{v}_{2}^{\text {stiff }}\left(l, l^{\prime}\right)\right\}
\end{aligned}
$$

\section{Orientational distribution function}

For the orientational distribution function, we have assumed the Onsager trial form [10]:

$$
f_{l}(\cos \theta)=\frac{\alpha_{N, l}}{4 \pi \sinh \alpha_{N, l}} \cosh \left(\alpha_{N, l} \cos \theta\right)
$$

where $\alpha_{N, l}$ is a parameter related to the degree of orientational order $\left(\alpha_{N, l}>0\right.$ for calamitics and $\alpha_{N, l}=0$ in the isotropic phase). This distribution function is normalized as $\int_{0}^{\pi} 2 \pi \sin (\theta) d \theta f_{l}(\theta) \simeq 1$. The parameter $\alpha_{N, l}$ is uniquely related to the order parameter $\left\langle P_{2}\right\rangle_{l}$, defined as:

$$
\left\langle P_{2}\right\rangle_{l} \equiv \int_{0}^{\pi} f_{l}(\theta) P_{2}(\cos \theta) 2 \pi \sin (\theta) d \theta \approx 1-3 / \alpha_{N}
$$

where $P_{2}$ is the second-order Legendre polynomial and the approximation holds in the limit $\alpha_{N} \rightarrow \infty$.

For simplicity, in our calculations the length dependence of the orientational distribution function, i.e. of the parameter controlling the width of the orientational distribution function, Eq. (37), was disregarded: $\alpha_{N, l}=\alpha_{N}$ for any $l$ value [4]. This means that we can approximate $\bar{\rho}(l, \boldsymbol{\Omega})=\nu(l) f(\cos \theta)$, with the two factors independent of each other.

\section{Orientational entropy}

For the contribution of semi-flexible polymers of length $l$ to the orientational entropy two limiting expressions have been proposed, which for the Onsager trial function, Eq. (37), takes the form [11]:

$$
\sigma_{o}(l)=\left\{\begin{array}{cc}
\ln \alpha_{N}-1+\frac{\alpha_{N}-1}{6 l_{p}} l & \left(l \ll l_{p} / \alpha_{N}\right) \\
\ln \left(\alpha_{N} / 4\right)+\frac{\alpha_{N}-1}{4 l_{p}} l & \left(l \gg l_{p} / \alpha_{N}\right)
\end{array}\right.
$$

We have written the orientational entropy of our system, which contains aggregates of all lengths, as the sum of contributions having the form of either one or the other of the two limiting expressions:

$$
\hat{\sigma}_{0}=\sum_{l=1}^{l_{0}^{\prime}-1} \nu(l)\left\{\left[\ln \alpha_{N}-1\right]+\frac{\alpha_{N}-1}{6 l_{p}} l\right\}+\sum_{l=l_{0}^{\prime}}^{\infty} \nu(l)\left\{\left[\ln \frac{\alpha_{N}}{4}+\frac{\alpha_{N}-1}{4 l_{p}} l\right\}\right.
$$

with $l_{0}^{\prime}$ as the boundary length between the two limiting cases. This value was determined as explained in ref. [5]. Since the length $l_{0}$ introduced in Eqs. (35) and (36) discriminates the regimes of stiff chains $\left(l<l_{0}\right)$ and fully flexible chains $\left(l \geq l_{0}\right)$ we found appropriate to set in our calculations $l_{0}=l_{0}^{\prime}$.

\section{Semi-flexible chains}

It is convenient to assume for the orientational distribution function and the chain length distribution a given functional form, in terms of a number of parameters which are used as variational parameters in the minimization of the free energy. As proposed in ref.[4], we have assumed that the chain length distribution $\nu(l)$ is exponential with an average 
chain length $M$ :

$$
\nu(l)=\rho M^{-(l+1)}(M-1)^{l-1}
$$

where

$$
M=\frac{\sum_{l=1}^{\infty} l \nu(l)}{\sum_{l=1}^{\infty} \nu(l)}
$$

\section{Determination of the stable phase and of the $\mathrm{I} \mathrm{N}^{*}$ phase coexistence}

The free energy Eq. (21) of the untwisted $\mathrm{N}$ phase is a functional of the chain length distribution of aggregates, $\nu(l)$ and of the orientational distribution function $f_{l}(\cos \theta)$. Assuming the chain length distribution reported in Eq. (41) and using Eq. (37) with $\alpha_{N}$ independent of the aggregate length, the free energy in the untwisted $\mathrm{N}$ phase, Eq. (21), is expressed as:

$$
\begin{aligned}
\frac{F_{0}}{k_{B} T V} & =\hat{\sigma}_{o}-\rho \frac{\Delta F_{b}}{k_{B} T}\left(1-M^{-1}\right)+\eta(\phi)\left[B\left(\alpha_{N}\right) X_{0}^{2}+\frac{v_{m} k\left(\alpha_{N}\right)}{M}\right] \rho^{2}+ \\
& +\frac{\rho}{M}\left[\ln \left(\frac{v_{m} \rho}{M}\right)-1\right]-\rho \ln M+\rho \ln (M-1) \frac{M-1}{M}
\end{aligned}
$$

This free energy is a function of the parameters $\alpha_{N}$ and $M$, which control the orientational order and the chain length distribution, respectively. The equilibrium state at given values of the temperature T and monomer density $\rho$ is obtained by minimization of the free energy with respect to these parameters. The value $\alpha_{N}=0$ corresponds to the isotropic phase; for $\alpha_{N} \neq 0$ the stable phase is the cholesteric.

The I-N* phase coexistence is determined by minimizing the free energy of the system with respect to the parameter $\alpha_{N}$, Eq. (37) and to the average chain length $M$, under the conditions of mechanical and chemical equilibrium between the two phases. The following set of equations have to be satisfied:

$$
\begin{aligned}
\frac{\partial F^{I}\left(\rho_{I}, M_{I}\right)}{\partial M_{I}} & =0 \\
\frac{\partial F^{N}\left(\rho_{I}, M_{N}, \alpha_{N}\right)}{\partial M_{N}} & =0 \\
\frac{\partial F^{N}\left(\rho_{I}, M_{N}, \alpha_{N}\right)}{\partial \alpha_{N}} & =0 \\
P_{I}\left(\rho_{I}, M_{I}\right) & =P_{N}\left(\rho_{N}, M_{N}, \alpha_{N}\right) \\
\mu_{I}\left(\rho_{I}, M_{I}\right) & =\mu_{N}\left(\rho_{N}, M_{N}, \alpha_{N}\right)
\end{aligned}
$$

where the indices I and $\mathrm{N}$ refer to the isotropic and the cholesteric phase, respectively.

\section{PARAMETERIZATION FOR DICKERSON DODECAMERS}

Calculations were performed for DD dodecamers with the self-complementary sequence CGCGAATTCGCG [12]. We used the same model of linear aggregates described in [13] and the coarse-grained representation proposed in [14]. The crystal coordinates of DD dodecamers were used and the end-to-end stacking geometry found in crystals was assumed (PDB entry 1BNA). 


\section{Scaling laws for excluded volume terms of stiff chains}

To obtain a manageable form of the free energy $F_{0}^{e x c l}$, of the chiral strength and of the twist elastic constant, it is convenient to express the excluded volume contributions $v_{i}^{\text {stiff }}$ appearing in Eqs. (21), (35) and (36) in polynomial forms with respect to the scaled length $l$. We calculated such terms by numerical integration of Eqs. (15)-(17) and the results are shown in Fig. (1).
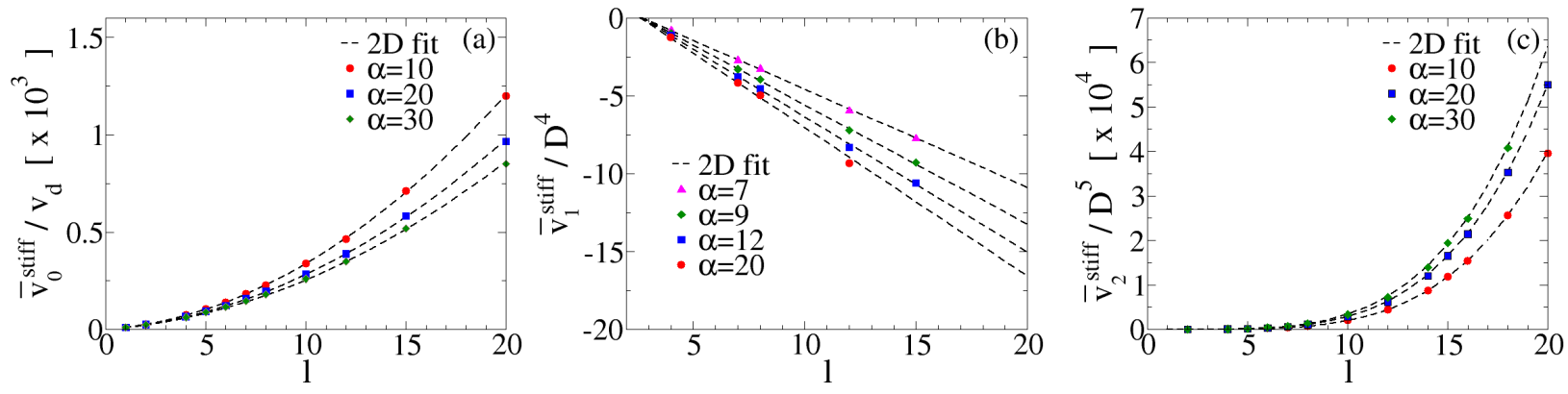

FIG. 1: Excluded volume terms calculated for different values of the orientational parameter $\alpha_{N}$ as a function of chain length $l$ for stiff aggregates (symbols), together with two-dimensional fits (solid lines) to numerical data using the scaling laws in Eqs. (45)-(47): (a) $v_{0}^{\text {stiff }} / v_{m}$ (b) $v_{1}^{\text {stiff }} / D^{4}$ and (c) $v_{2}^{\text {stiff }} / D^{5}$.

We found that a good modeling of our numerical data is provided by the following scaling laws:

$$
\begin{aligned}
\bar{v}_{0}^{\text {stiff }}\left(l, l^{\prime}, \alpha_{N}\right) & =2 \frac{\pi}{4} D^{3}\left(\eta_{1}+\frac{\eta_{2}}{\alpha_{N}^{1 / 2}}+\frac{\eta_{3}}{\alpha_{N}}\right) X_{0}^{2} l l^{\prime}+2 v_{m} k_{N}^{H C}\left(\alpha_{N}\right) \frac{l+l^{\prime}}{2} \\
\bar{v}_{1}^{\text {stiff }}\left(l, l^{\prime}, \alpha_{N}\right) & =2\left[\left(a_{1}+\frac{a_{2}}{\alpha_{N}^{1 / 2}}+\frac{a_{3}}{\alpha_{N}}\right)+\left(b_{1}+\frac{b_{2}}{\alpha_{N}^{1 / 2}}+\frac{b_{3}}{\alpha_{N}}\right) \frac{l+l^{\prime}}{2}\right] \\
\bar{v}_{2}^{\text {stiff }}\left(l, l^{\prime}, \alpha\right) & =2 l l^{\prime}\left[l l^{\prime} \frac{\pi}{4} D^{3} X_{0}^{2}\left(\frac{c_{1}}{\alpha_{N}^{1 / 2}}+\frac{c_{2}}{\alpha_{N}}+\frac{c_{3}}{\alpha_{N}^{3 / 2}}\right)+\xi v_{m} k_{N}^{H C}\left(\alpha_{N}\right) \frac{l+l^{\prime}}{2}\right]
\end{aligned}
$$

where $v_{m}, D, X_{0}$ are the volume, diameter and aspect ratio of a single DD respectively and the term $2 v_{m} k_{N}^{H C}\left(\alpha_{N}\right)$ is the end-midsection contribution to the excluded volume of two hard cylinders of volume $v_{m}$ and aspect ratio $X_{0}$ (see ref. [5] for more details). In Eqs. (45)-(47), $\xi, \eta_{k}, a_{k}, b_{k}$ and $c_{k}$ with $k=1,2,3$ are parameters that can be obtained by a fit of $v_{i}^{\text {stiff }}(l, l, \alpha)$ to the numerical data as shown in fig. (1). Note that the fitting procedure is two-dimensional in that the fitting function depends on the two variables $l$ and $\alpha$. The scaling law for $v_{0}^{\text {stiff }}$ has the same form used in ref. [5].

\section{Geometric parameters}

The geometric parameters appearing in Eqs. (45)-(47), i.e diameter $D$, aspect ratio $X_{0}$ and volume $v_{m}$ of a DD, were estimated as follows. Considering an aggregate of length $l=10$, its volume, $v_{10}=10 v_{m}$, was calculated as the volume enclosed by the surface drawn by a sphere with radius equal to the size of half a base pair $\left(r_{\text {probe }}=1 \mathrm{~nm}\right)$ rolling over it [15]. The parameters $X_{0}$ and $D$ were determined by mapping the volume, $v_{10}$, and the average excluded volume in the isotropic phase, $\bar{v}_{0}^{\text {excl }}(10,10)$, calculated for the aggregate into the volume and excluded volume for an equivalent cylinder. An explicit expression for the latter is available [10]. Thus, the following algebraic equations were solved (with $X_{0}$ and $D$ as unknown):

$$
\left\{\begin{aligned}
v_{10} & =10 \frac{\pi}{4} D^{3} X_{0} \\
\bar{v}_{0}^{e x c l}(10,10) & =\frac{\pi^{2}}{8} D^{3}+2 \cdot 10^{2}\left(3 \frac{\pi}{8}+\frac{\pi^{2}}{8}\right) X_{0} D^{3}+10^{3} \frac{\pi}{2} X_{0}^{2} D^{3}
\end{aligned}\right.
$$


Both volume and excluded volume of aggregates were calculated using the coarse grained model. The values obtained for the $D D$ are: $v_{m}=11.65 \mathrm{~nm}^{3}, X_{0}=1.94$ (i.e a length $L=3.82 \mathrm{~nm}$ ) and $D=1.97 \mathrm{~nm}$. Latter values are in line with canonical B-DNA structure with $D=2 \mathrm{~nm}$ and a $L=N_{b} / 3=4 \mathrm{~nm}$, where $N_{b}$ is the number of base pairs [16].

\section{Stacking free energy}

For the term $\Delta F_{b}$ appearing in Eq. (4) we assumed the form proposed in ref. [5], where the bonding entropy and stacking energy are in first approximation assumed to be $T$-independent:

$$
\Delta F_{b}=k_{B} T\left[\ln \left(\frac{4 D^{3}}{v_{m}}\right)-17.6769+\frac{4559 K}{T}\right]
$$

Although this choice is rather crude it provides a reasonable estimate for the stacking free energy at a standard concentration $1 \mathrm{M}$ of DNADs and $T=293 \mathrm{~K}$ ( i.e. under standard conditions) of $-0.5 \mathrm{kcal} / \mathrm{mol}$.

* Electronic address: cristiano.demichele@roma1.infn.it

present address: BMSSI, Uni. Lyon 1 / CNRS UMR 5086, IBCP, 7 passage du Vercours, 69367 Lyon, France

[1] I. Dierking, Textures of Liquid Crystals, Wiley-VCH, 2003.

[2] S. Chandrasekhar, Liquid Crystals, Cambridge University Press, Cambridge, UK, 2nd edn., 1992.

[3] D. A. McQuarrie, Statistical Mechanics, University Science Books, Sausalito, CA, 2000.

[4] C. De Michele, T. Bellini and F. Sciortino, Macromolecules, 2012, 45, 1090-1106.

[5] C. De Michele, L. Rovigatti, T. Bellini and F. Sciortino, Soft Matter, 2012, 8, 8388-8398.

[6] J. Parsons, Phys. Rev. A, 1979, 19, 1225.

[7] S. Lee, J. Chem. Phys., 1987, 87, 4972.

[8] S. Lee, J. Chem. Phys., 1988, 89, 7036.

[9] K. T. Nguyen, F. Sciortino and C. De Michele, Langmuir, 2014, 30, 4814-4819.

[10] L. Onsager, Ann. N.Y. Acad. Sci., 1949, 51, 627-659.

[11] T. Odijk, Macromolecules, 1986, 19, 2313-2329.

[12] R. Wing, H. Drew, T. Takano, C. Broka, S. Tanaka, K. Itakura and R. E. Dickerson, Nature (London), 1980, 287, $755-758$.

[13] E. Frezza, F. Tombolato and A. Ferrarini, Soft Matter, 2011, 7, 9291-9296.

[14] F. Tombolato and A. Ferrarini, J. Chem. Phys., 2005, 122, 054908.

[15] M. F. Sanner, A. J. Olson and J. C. Spehner, Biopolymers, 1996, 38, 305.

[16] M. Nakata, G. Zanchetta, B. D. Chapman, C. D. Jones, J. O. Cross, R. Pindak, T. Bellini and N. A. Clark, Science, 2007, 318, 1276-1279. 